\title{
La terminologie de parenté au Ladakh (Leh)
}

Kinship terminology in Ladakh (Leh)

\section{Patrick Kaplanian}

\section{(2) OpenEdition}

\section{Journals}

Édition électronique

URL : https://journals.openedition.org/emscat/2634

DOI : $10.4000 /$ emscat.2634

ISSN : 2101-0013

Éditeur

Centre d'Etudes Mongoles \& Sibériennes / École Pratique des Hautes Études

\section{Référence électronique}

Patrick Kaplanian, «La terminologie de parenté au Ladakh (Leh) », Études mongoles et sibériennes, centrasiatiques et tibétaines [En ligne], 46 | 2015, mis en ligne le 10 septembre 2015, consulté le 13 juillet 2021. URL : http://journals.openedition.org/emscat/2634; DOI : https://doi.org/10.4000/ emscat.2634

Ce document a été généré automatiquement le 13 juillet 2021.

(c) Tous droits réservés 


\section{La terminologie de parenté au Ladakh (Leh)}

Kinship terminology in Ladakh (Leh)

Patrick Kaplanian

1 Depuis les travaux du Prince Pierre de Grèce $(1956,1963)$ et ceux de Grist $(1977$, p. 46 et surtout 1979, pp. 218-244) peu de choses ont été écrites sur la nomenclature de parenté. Je pense que le moment est venu d'étoffer le dossier.

\section{Les termes d'adresse à Leh}

\section{Le noyau de base}

2 En matière de termes d'adresse le premier schéma, le plus simple, ne semble pas à première vue relever franchement de la terminologie de parenté, puisqu'il s'agit de termes utilisés pour s'adresser à n'importe qui, depuis l'inconnu qu'on croise dans la rue jusqu'à ses proches. Il s'agit de neuf termes (fig. 1).

Figure 1

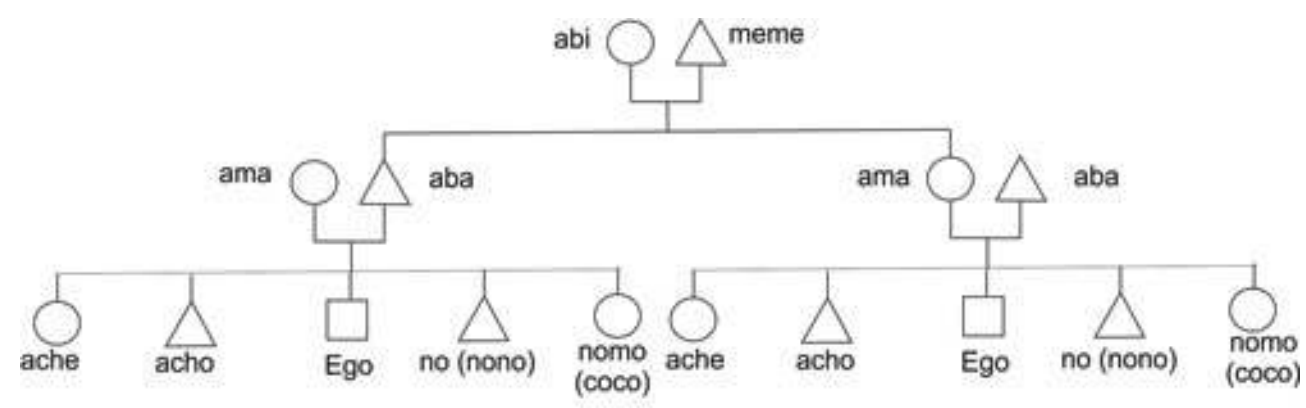

$3 \quad A b i$ (a-phyi), " grand-mère », $\mathrm{FM}, \mathrm{MM}^{1}$ et toute femme de la génération Ego +2 ou plus. Meme (mes-mes) « grand-père », FF, MF et tout homme de la génération Ego + 2 ou plus. $A b a(a-p h a)$ « père », F et tout homme de la génération Ego +1. 
Ama $(a-m a)$ « mère », $\mathrm{M}$ et toute femme de la génération Ego + 1.

$A c o(a-j o)$ « frère aîné », eB et tout homme de la génération d'Ego plus âgé qu'Ego.

Ache (a-che) « sœur aînée ", eZ et toute femme de la génération d'Ego plus âgée qu'Ego.

Nono (no-no) « petit-frère ", yB et tout homme de la génération d'Ego un peu plus jeune

qu'Ego. Ou encore d'une génération inférieure. Nono était le terme honorifique pour no

(no). Maintenant il s'emploie couramment concurremment à no qui est beaucoup plus rare.

Nomo (no-mo) « sœur cadette », $\mathrm{yZ}$ et toute femme de la génération d'Ego plus jeune que lui, ou encore d'une génération inférieure.

Enfin coco (jo-jo) s'emploie parfois pour appeler une toute petite fille (coco désigne aussi la poupée). Ici aussi il s'agit d'un terme honorifique passé dans le langage courant.

4 Ce « noyau de base » des termes d'adresse appelle trois réflexions :

a) La première est l'existence d'une triple graduation vers le haut : l'aîné (aco ou ache), la génération d'Ego + 1 (aba ou ama), la génération d'Ego + 2 (meme ou abi). Vers le bas il n'y a qu'un degré, nono ou nomo (et à la rigueur un peu plus si l'on tient compte de coco). Un vieil homme emploiera nono pour un plus jeune que lui, qui pourrait être son cadet, son fils, son petit-fils, voire plus (son SSS ou son SSSS).

b) Tous les Ladakhi interrogés disent que nono et coco sont des termes honorifiques pour no et nomo. En pratique en ce qui concerne nono il est systématiquement employé pour un plus jeune et pas seulement pour les «nobles ", skutraks (sku-drag). Par contre on emploiera effectivement coco à la place de nomo pour une plus jeune «noble " réservant coco dans le vocabulaire courant à une très petite fille.

On peut se poser la question de l'emploi courant actuel de ce vocabulaire utilisé jadis pour les seuls cadets nobles car si le vocabulaire honorifique se délite en matière de cadets, il ne se délite pas en matière d'aînés. On dira kaga au lieu d'aco et shema au lieu d'ache pour un(e) noble.

En fait cela montre que le Ladakh est une société très hiérarchisée et qu'elle le reste dans les mentalités. L'existence de trois degrés vers le haut, et le maintient d'une stricte différence entre aînés, nobles ou pas, s'oppose à l'existence d'un seul degré vers le bas et à la disparition, toujours vers le bas, d'une distinction entre le vocabulaire honorifique et le vocabulaire « roturier $»^{2}$.

c) Comme je viens de le dire, ces termes dépassent le cercle étroit de la parenté. Un père ou une mère, pourra dire nomo, nono à son jeune frère, à son fils à sa fille, à ses petits-enfants, à ses neveux, à ses petits-neveux et même en dehors de sa famille puisque nomo, nono s'emploient pour désigner n'importe qui de la génération d'Ego - 1 ou d'Ego mais d'un âge inférieur à Ego, au point qu'on peut se demander s'il s'agit véritablement d'une nomenclature de parenté. $\mathrm{Si}$, en effet, aba désigne toute personne de la génération d'Ego +1 s'agit-il d'une métaphore par laquelle on assimile tout homme en âge de l'être à un père, ou s'agit-il tout simplement d'un mot qui veut dire « homme de la génération d'Ego +1 » et qui s'emploie pour le père parce que celui-ci en fait partie? Rien ne permet de trancher, pour le moment, entre les deux termes de l'alternative.

\section{Les degrés de parenté}

5 Pour répondre à cette dernière question nous allons considérer les degrés de parenté. Le mot rtsima (rtsib-ma) veut dire côte au sens de l'os de la poitrine. Il désigne aussi les degrés de parenté. Comment calcule-t-on les degrés de parenté ? Entre deux germains 
(frère et sœur $\mathrm{E}$ et $\mathrm{F}$ ) il y a un degré de parenté. Entre un parent et un enfant (A et E/F) aussi (fig. 2) : il y a un degré entre un frère et une sœur parce qu'il n'y a qu'une génération qui les sépare de l'ancêtre commun. Donc si l'on passe à deux générations entre un grand-père et un petit-fils ( $A$ et $G / B$ par exemple), il y a deux degrés de parenté, donc entre deux cousins germains ( $G$ et $B$ ) aussi. Donc entre A et B par exemple il y a deux degrés de parenté, et entre $B$ et $C$ ou $D$ aussi. On voit donc :

a) que le calcul des rtsima est bilatéral, peu importe si l'ancêtre commun est un homme ou une femme,

b) que le calcul des degrés de parenté se fait en remontant l'arbre généalogique ${ }^{3}$.

6 Maintenant voyons le cas de $\mathrm{B}$ et $\mathrm{C}$. Il y a deux générations entre $\mathrm{B}$ et $\mathrm{C}$, donc deux degrés de parenté. Or il peut très bien se faire que $C$ et $B$ soient deux personnes d'âge assez proche. Si elles ignorent leur lien de parenté, ce qui est fréquent, elles vont s'appeler, si ce sont des femmes, ache et nomo, sœur aînée et sœur cadette, selon leurs âges respectifs. Mais si l'arbre généalogique est connu, alors $\mathrm{C}$ appellera $\mathrm{B} a b i$, grand'mère, et $B$ appellera $C$ tshamo. Il m'est arrivé effectivement de voir une femme d'une soixantaine d'années en appeler une autre de sa génération abi. L'explication qui m'a été donnée est celle qui précède. On voit donc que la notion de "savoir » est très importante. Lorsqu'on sait on emploie les termes de parenté précis, lorsqu'on ne sait pas on utilise le noyau de base ou des termes de parenté vagues ${ }^{4}$. La conclusion de tout cela c'est que aba, ama, abi, meme, etc. sont bien des termes de parenté, utilisés par défaut lorsque le lien de parenté est inconnu. Il en est de même des moines. Si tel moine est le jeune frère de mon père je l'appellerai agu et non pas azhang (cf. infra $)^{5}$.

Figure 2

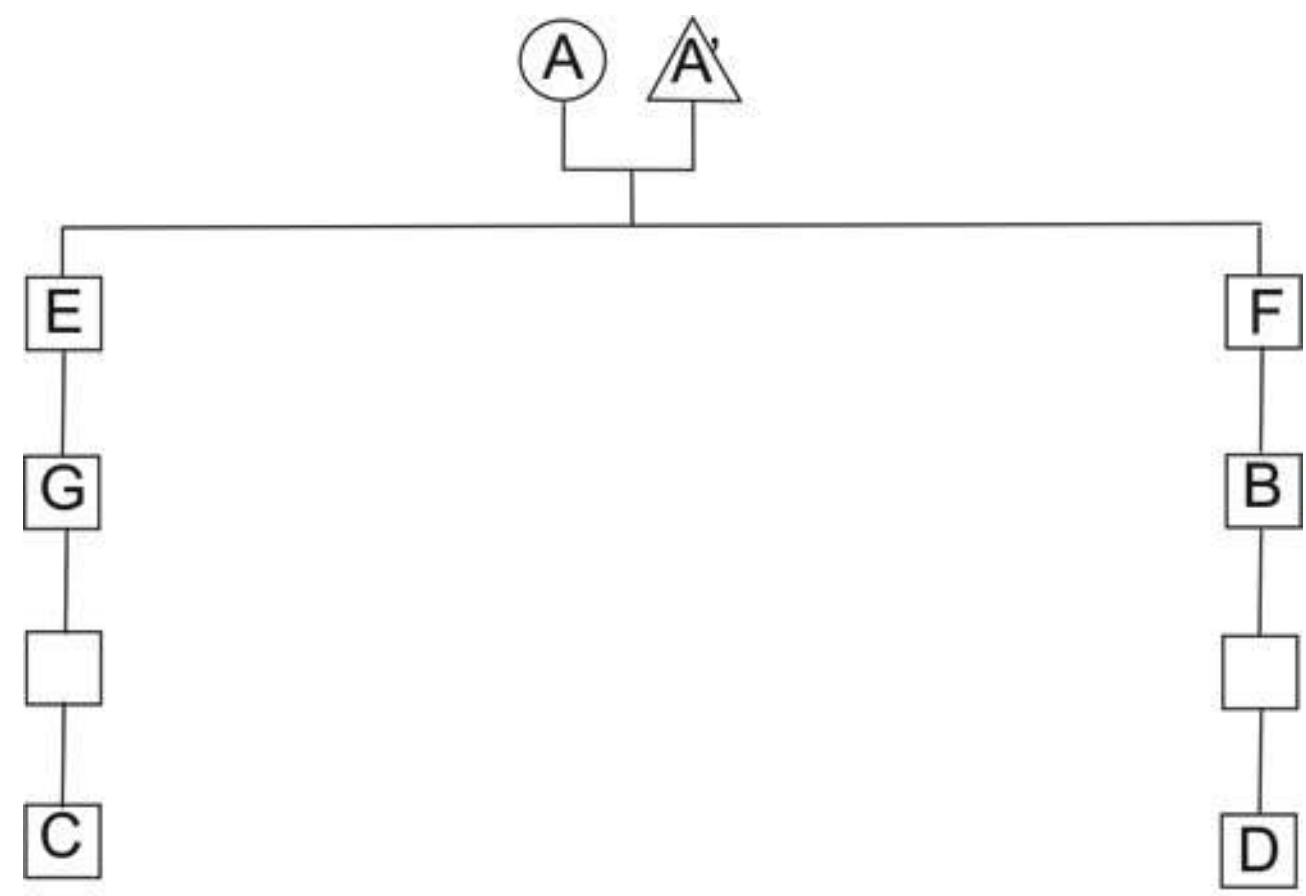




\section{Le tableau complet des termes d'adresse}

\section{Les consanguins}

Génération Ego + 2. Pas de changement. Il n'y a que deux termes, abi et meme.

Figure 3

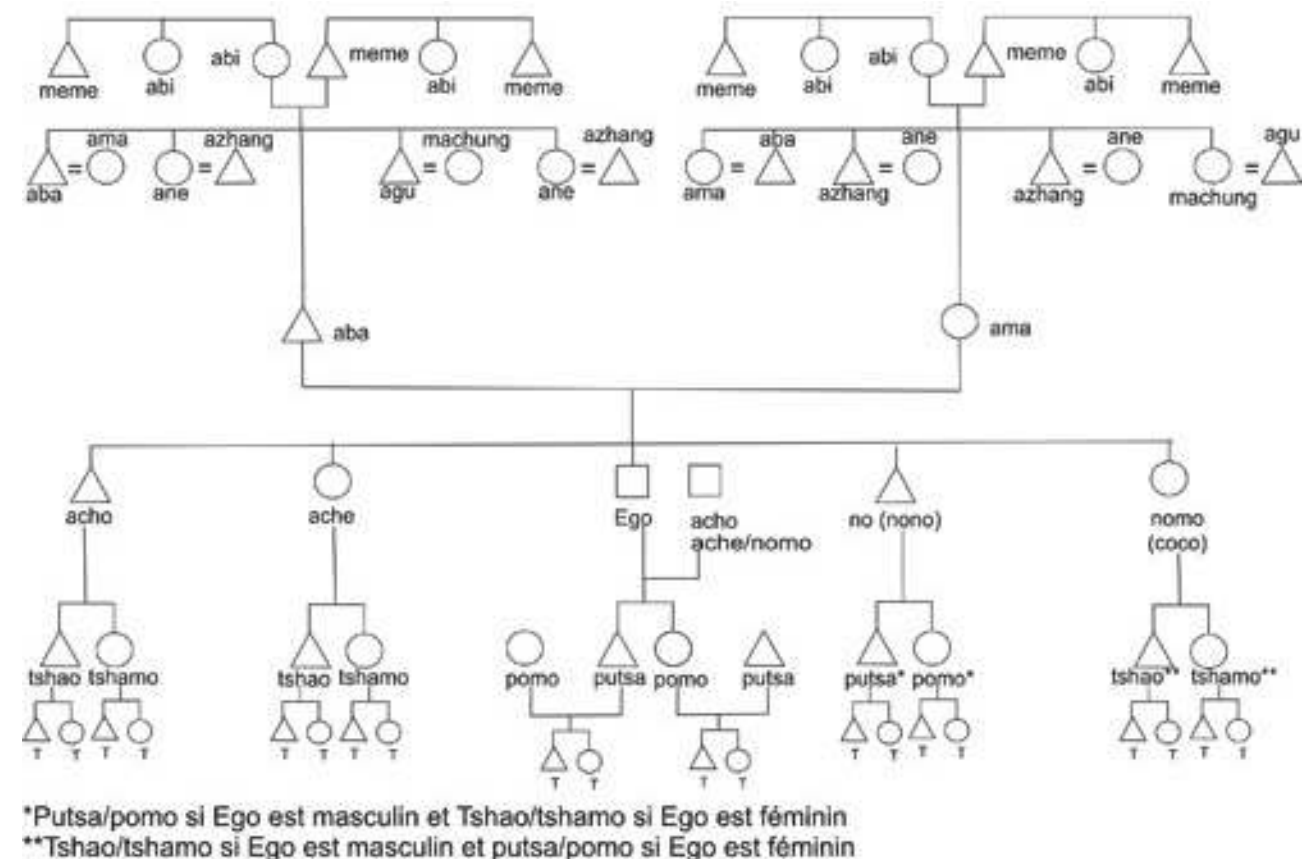

Génération Ego + $1: a b a(\mathrm{~F})$ et ama $(\mathrm{M})$ désignent aussi FeB et MeZ. FZ est ane qu'elle soit plus âgée ou plus jeune que le père d'Ego et $\mathrm{MB}$ est azhang qu'il soit plus âgé ou plus jeune que la mère d'Ego. FyB est agu et MyZ est machung.

Cette nomenclature de la génération Ego + 1 est la plus originale. Je vais longuement y revenir. Signalons tout de suite que les termes sont les mêmes pour les alliés. La femme de aba est ama et réciproquement, celle d'azhang est ane et réciproquement, celle d'agu est machung et réciproquement. Pour plus de détails voir plus loin.

Génération d'Ego. Pas de changement. Les quatre termes sont nono, aco, nomo et ache.

Génération Ego - 1 : fils (S) : putsha (bu-tsha) ; fille (D) : pomo (bu-mo). Pour les neveux pomo/putsha ou tshao/tshamo (tsha-bo/tsha-mo). Pour plus de détails voir plus bas. Comme déjà dit les termes nono, nomo peuvent être employés. Comme en français et dans beaucoup de langues les mots putsha (bu-tsha); fille (D) : pomo (bu-mo) peuvent avoir le sens général de garçon et fille sans référence à la parenté : « $i$ pomo demo duk : cette fille est jolie $»^{6}$.

Génération d'Ego - 2 : tshao/tshamo dans tous les cas (petits-enfants et petits-neveux). Idem au-delà (Ego - 3, etc.). Nous retrouvons à cette génération la simplification en deux termes que nous avions à la génération Ego + 2. Les deux seuls critères sont le sexe et la génération.

Donc apparaissent, concurremment à no/nono et nomo/coco, les termes de pomo (bu-mo, fille), putsha (bu-tsha, fils), tshao (tsha-bo ; petit-fils ou neveu) et tshamo (tsha-mo petitefille ou nièce). Mais ces quatre termes s'emploient concurremment à no/nono et nomo/ coco. 
12 Il y a toujours trois degrés vers le haut. Vers le bas cela semble se complexifier. Il y a au moins deux degrés. Les cadets, puis la génération Ego - 1. Mais les termes tshao/tshamo sont transgénérationels puisqu'ils s'emploient aussi bien pour certains «neveux » et « nièces » que pour les petits-enfants.

13 Le $2^{\mathrm{e}}$ schéma met donc en évidence :

a) la simplicité des niveaux de Ego +2 et Ego-2: tous les grands parents, tous les grands-oncles et grands-tantes, par alliance ou pas, et tous les gens de la génération d'Ego + 2, s'appellent abi et meme. Tous les petits-fils et petits-neveux s'appellent tshao et tshamo.

b) La même simplicité relative au niveau d'Ego. Tous les frères et sœurs, et tous les cousins, et toutes les personnes de la génération d'Ego consanguins, utérins et alliés, sont soit no/nono//aco soit nomo/coco//ache. Aux deux critères du sexe et de la génération s'ajoute celui de l'âge relatif.

On peut déjà faire une première synthèse. Au niveau d'Ego le système n'est pas de type eskimo puisqu'il ne distingue pas les germains (frère et sœur) des cousins, mais de type hawaïen. C'est un système dit "générationnel » dans la mesure où tous les frères et cousins, sœurs et cousines sont désignés par le même terme. Mais il faut prendre en compte par contre la différenciation entre cadet et aîné qui n'existe pas dans le système strictement hawaïen qui n'emploie qu'un terme pour frère ou cousin aîné ou cadet et un terme pour sœur et cousine aînée ou cadette ${ }^{7}$.

15 Il s'agit donc d'un système à tendance générationnelle ou hawaïenne mâtiné de l'opposition aîné/cadet sauf pour les termes permettant de s'adresser à la génération Ego +1 qui forment un ensemble beaucoup plus compliqué. En résumé les critères sont : Ego $+2:$ sexe et génération.

Ego +1 : Sexe, génération, âge relatif par rapport au père ou à le mère d'Ego.

Ego : sexe, génération, âge relatif par rapport à Ego.

Ego-1: sexe, génération, mais il faut compter avec deux termes transgénérationels tshao/tshamo.

Ego-2: sexe, génération, mais il faut compter avec deux termes transgénérationels tshao/tshamo.

Il restera à voir le niveau Ego + 1 et le problème des termes transgénérationels tshao/ tshamo.

\section{Les affins les plus proches}

En ce qui concerne la génération d'Ego nous avons deux règles contradictoires. Selon la première le mari de ma sœur aînée (ache) est frère aîné (acho) et la femme de mon frère aîné (acho) est sœur ainée (ache). Le mari de tshao est tshamo et réciproquement. La référence est le parent le plus proche.

Selon la seconde pour s'adresser aux maris de sœurs et femmes de frères, on ne considère que l'âge relatif par rapport à soi-même. Si par exemple ma petite sœur (nomo) a un mari plus âgé que moi, je l'appellerai aco, et s'il est plus jeune que moi, je l'appellerai nono.

Les choses ne sont pas claires. Ainsi j'ai entendu une fois un homme d'une cinquantaine d'années, s'adressant à un couple. Le destinateur d'une cinquantaine d'années était plus jeune que le mari du couple (une soixantaine d'années) mais plus âgé que la femme (une quarantaine d'années). Il les appelait acho et ache, et de me donner la règle « la 
femme de acho est ache ». Je lui demandai alors comment il aurait fait s'il la connaissait avant son mariage. Il me répondit qu'effectivement elle serait passée de nomo à ache et que, encore aujourd'hui, s'il la croise seule sans son mari dans le bazar elle redevient nomo.

Il est à noter que même mari et femme s'appellent entre eux aco/ache/nono/nomo. Si l'homme est le plus âgé ce qui est le cas le plus fréquent il va appeler sa femme ache en fonction de la première règle et nomo en fonction de la seconde.

Par contre nous allons voir qu'à toutes les autres générations, par exemple à la génération Ego + 1 c'est la première règle qui est appliquée et de façon stricte, ce qui s'explique assez facilement puisque de toute façon, sauf cas exceptionnel rare, ils sont nettement plus âgés qu'Ego, et que donc il ne peut y avoir conflit entre les deux règles.

\section{Les autres affins}

En ce qui concerne les frères et sœurs du conjoint d'Ego et d'une façon générale tous les parents du conjoint d'Ego, ici encore nous avons affaire à deux règles contradictoires. La première est : «quelle que soit la façon dont j'appelle mes parents, ma femme les appelle de la même façon. Quelle que soit la façon dont ma femme appelle ses parents, je les appelle de la même façon ». J'appelle cette règle, la règle d'identité d'appellation entre mari et femme. Et par ailleurs nous avons une fois de plus la règle de l'âge réel.

\section{Les germains de père et mère}

Nous allons maintenant nous intéresser aux termes de la génération Ego + 1 que sont : azhang, machung, agu, ane. Tout d'abord, il s'agit de termes utilisés, eux aussi, quelquefois au-delà du cercle familial, quoiqu'à Leh, même si cet emploi est admis théoriquement, il est assez rare, sauf en ce qui concerne azhang ou ane utilisés pour un moine ou une nonne. Dessinons cette partie du tableau pour mieux l'analyser (fig. 4) :

Figure 4

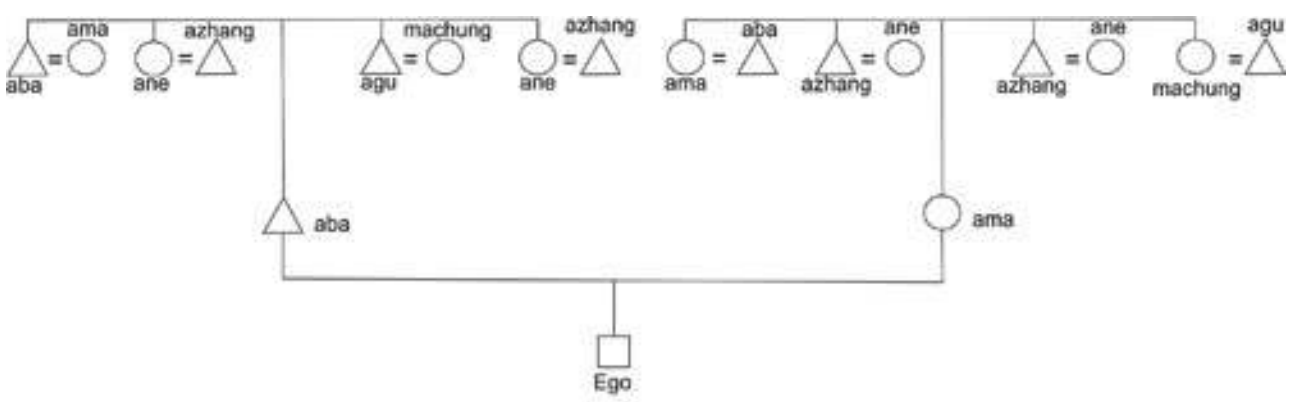

On voit que pour les oncles paternels d'Ego, les oncles parallèles, on distingue entre l'oncle aîné paternel d'Ego ( $\mathrm{FeB}, a b a$, comme le père) et l'oncle cadet paternel d'Ego (FyB, agu). Par contre la sœur aînée du père (FeZ) et la sœur cadette du père (FyZ) s'appellent toutes les deux ane. À l'opposé en ce qui concerne les oncles et tantes du côté de la mère c'est le contraire. On ne distingue pas les frères de la mère (MeB et MyB), qui sont tous deux azhang, l'aîné et le cadet, et on distingue les sœurs, l'aînée (MeZ) étant ama (comme la mère) et la cadette (MyZ) étant machung (=ama chumung: petite mère). 
critères de classification Kroeber $^{8}$ distingue huit types de relations possibles, dont la différence d'âge entre individus au sein d'une même génération qui au Ladakh existe donc pour les générations Ego et Ego +1 . Lowie ${ }^{9}$ a voulu raffiner le $2^{\mathrm{e}}$ critère de Kroeber, c'est-à-dire la différence entre relation de type linéaire (père, mère) et de type collatéral (oncle, tante). Il distingue quatre cas :

I) Oncles et tantes sont assimilés terminologiquement au père et à la mère. C'est à nouveau le système hawaïen (générationnel).

II) Le frère du père est assimilé au père, alors que le frère de la mère est désigné par un terme spécifique et réciproquement la sœur de la mère est assimilée à la mère et la sœur du père est désignée par un terme spécifique. C'est le système à bifurcation et à assimilation (bifurcate merging) aussi appelé iroquois.

III) Le frère de la mère et le frère du père sont distincts du père et de la mère et l'un de l'autre. Idem pour les tantes. C'est le système à bifurcation et à lignes (bifurcate collateral).

IV) Notre système (français et anglais entre autres) : des termes pour père et mère, et un terme pour sœur de père et sœur de mère (tante/aunt), un autre pour frère de père et frère de mère (oncle/uncle).

Cette classification est déjà ancienne mais elle garde encore aujourd'hui tout son intérêt surtout d'un point de vue pédagogique. Elle est d'ailleurs fréquemment citée ${ }^{10}$.

Quoiqu'il en soit le schéma ladakhi ne correspond à aucun des types décrits par Lowie. C'est un mélange du type II et du type III. Les frères aînés seulement du père sont appelés aba comme le père et les sœurs aînées seulement de la mère sont appelées ama comme la mère comme dans le type II. Le jeune frère du père porte un nom spécifique (agu) et la jeune sœur de mère aussi (machung, c'est-à-dire en fait ama chumung, " petite-mère ») comme dans le type III. Par contre on retombe bien dans le type II de Lowie avec un terme spécifique pour les sœurs de père aînées ou cadettes (ane) et les frères de mère aînés ou cadets (azhang).

On peut se poser la question de la non-mention par Lowie de ce mélange : différence entre aîné et cadet dans une autre génération que celle d'Ego. Lowie ignore complètement ce critère dans la génération d'Ego + 1 à laquelle il consacre son article. Pourtant des exemples existent et le Ladakh est loin d'être un cas unique. En Chine la terminologie établit quatre types de distinction suivant le sexe, l'âge relatif, la génération et la filiation. Là où le français ou l'anglais se contentent d'oncle/uncle le chinois dispose de cinq termes $1^{\circ}$ ) FeB, $2^{\circ}$ ) FyB, $3^{\circ}$ ) MB, $4^{\circ}$ ) FZH, $5^{\circ}$ ) $\mathrm{MZH}^{11}$. C'est à la fois plus simple et plus compliqué que le ladakhi. Plus compliqué parce qu'il y a encore plus de termes, mais plus simple en fin de compte parce que les règles sont claires: $\mathrm{FeB} \neq \mathrm{F} \neq$ FyB. À chaque position un mot.

Études mongoles et sibériennes, centrasiatiques et tibétaines, 46 | 2015 
C'est que le rajout de ce critère ${ }^{12}$ met par terre une théorie qui a fait fureur dans l'anthropologie anglo-saxonne de Tylor à Radcliffe-Brown en passant par Lowie et qui lie une terminologie de type iroquois à l'existence de groupes de filiation unilinéaires (GFU, unilineal descent groups), c'est-à-dire à l'exogamie. Selon la théorie de Tylor et Rivers, reprise par Lowie et Radcliffe-Brown, le fait d'appeler le FB comme le F et la MZ comme la $\mathrm{M}$ renvoie à la solidarité de groupe. L'idéologie est la consanguinité. Comme l'écrivait Tylor clan exogame et terminologie classificatoire (iroquoise) «sont en fait deux faces d'une seule et même institution $»^{13}$.

Or l'existence des termes agu et machung met tout cela par terre. Car machung appartient au même GFU qu'ama. Avant le mariage s'entend, et c'est bien de cela que parle Radcliffe-Brown. De même pour agu et aba. On objectera que dans le système traditionnel, un agu se faisait gendre (makpa) dans le cadre d'un mariage uxorilocal (voir Grist 1977, 1979; Phylactou 1989 ; Kaplanian 1981) ou moine et que dans l'un et l'autre cas il quittait le phaspun (le clan) et la maison (khangpa, khang-pa). Mais cela n'est déjà plus tout à fait le raisonnement de Radcliffe-Brown et obligerait à tenir compte non seulement du groupe (maison et phaspun) d'origine mais du groupe d'adoption.

Dès 1897 Kohler $^{14}$ émettait, à propos des Omaha, l'hypothèse que le même terme de parenté renvoyait à ce qu'il appelait le «mariage de groupe » (Gruppenehe). Avant de prendre une seconde épouse, un Omaha faisait part à la première du désir d'alléger sa tâche. Et celle-ci pouvait répondre, ou prendre les devants, et dire "Je souhaite que tu épouses ma nièce, [ou ma sœur ou ma tante], parce qu'elle et moi nous sommes une seule chair. » Et effectivement pour un locuteur masculin omaha WFZ = WZ = WZD. Un même terme désignerait donc les personnes que l'on peut épouser ensemble.

Cette hypothèse a fait fortune. Sapir a avancé que le lévirat et le sororat préférentiels ainsi que la polyandrie fraternelle et la polygynie sororale peuvent rendre inopérant le critère de la collatéralité et engendrer ainsi des terminologies de type à " assimilation par bifurcation » (bifurcate merging, le type II de Lowie, c'est-à-dire que le frère du père est appelé « père » et la sœur de la mère, « mère »). Et cette hypothèse a été reprise par Kirchhoff et d'autres ${ }^{15}$.

reprend cette théorie de Sapir et Kirchhoff et en fait un "théorème " (le théorème 2, p. 147 de l'édition française) qui s'exprime comme suit: "En régime de polygynie sororale, les termes désignant les parents de premier ordre [c'est-à-dire $F, M$, H, W, S, D, B, Z, cf. Murdock p. 106] tendent à s'appliquer par extension à leurs parents collatéraux en ligne féminine de même sexe et de la même génération. »

Mais ce qui est surtout original au Ladakh c'est que seuls les FeB et MeZ sont assimilés respectivement à $\mathrm{F}$ et $\mathrm{M}$. En ce qui concerne les $\mathrm{FyB}$ et $\mathrm{MyZ}$, le système est à bifurcation et à lignes (bifurcate collateral), le type III de Lowie où les oncles et les tantes paternels et maternels sont non seulement distingués de $\mathrm{M}$ et $\mathrm{F}$ mais entre eux. En fait loin de réfuter la théorie de Kohler, Sapir et autres Murdock, le fait que FyB soit différent de $\mathrm{FeB}=\mathrm{F}$ la renforce. Il suffit de se référer à l'ethnographie. La polyandrie, en effet, n'implique pas le mariage de tous les frères avec la même femme, mais uniquement des deux premiers, à la rigueur des trois premiers. C'est dit et redit par tous les ethnographes (voir par exemple Ramsay $1890^{16}$, Grist 1979, p. 230). Autrement dit le " vrai » père d'Ego n'est pas son père mais son FeB et réciproquement puisqu'il n'y a de cérémonie de mariage qu'avec l'aîné(e) (Grist 1977, 1979). D'ailleurs dans le mariage polyandre le père biologique reste inconnu. 
pakston cho ches. The first wife of a man is called his "changch pakston cho ches. The first wife of a man is called his "changchhen", if she has no children, or if he is rich enough to keep two families, he may marry a second wife, who is called his "changchhungan", and if she too is childless, or if he can afford to keep three families, he may marry a third wife, who is called his "yángchhungan". So long as these are alive, he may not marry a fourth wife. If one wife dies, another wife may be taken in her place. As a matter of fact, the Ladákis are much too poor to wish to support more than one wife in a family, and it is only the necessity for having children, who will one day relieve them of the "forced labour" duties attaching to the possession of land, that induces them to remarry. If a man and his brothers have got, in common, three living wives and yet not child, they may not get another wife, but may call in to their family circle another man, to be a husband to their wives ; this man is termed a "phortsak". If he too begets no child, still another man, called "phó-chhungan", may be called in. If he too is childless, the original husband and wife must resort to adoption " (pp. 133-134); l'information est reprise par Hanlon ${ }^{18}$.

Études mongoles et sibériennes, centrasiatiques et tibétaines, 46 | 2015 
41 Ce type de mariage paraît un peu surréaliste et je doute qu'il n'ait jamais existé, du moins poussé à son extrême. Il faudrait en effet, pour en arriver là, que toutes les femmes ou tous les hommes soient stériles ce qui statistiquement, vu le nombre de personnes impliquées, est peu probable. Il ne peut néanmoins pas avoir été inventé. La terminologie est trop précise et nous possédons deux témoignages. Cela veut dire que cette polygynie sororale/polyandrie fraternelle induite par le vocabulaire de la parenté existe au moins dans les mentalités. Il est vrai que Ramsay n'indique pas que ces femmes sont sœurs ${ }^{19}$.

42 Les sœurs du père d'Ego (FZ) ne peuvent être potentiellement mère d'Ego, et elles portent toute la même adresse, ane, sans distinction d'aînée ou de cadette. De même les MB (azhang). Et l'époux d'azhang est ane et réciproquement. Ils ne font ni partie de la maison, ni du clan (phaspun, pha-spun).

\section{Moines et nonnes}

43 a) Azhang et ane s'emploient comme termes d'adresse pour les moines et les nonnes. Ces termes désignent ceux qui ne peuvent être potentiellement père ou mère d'Ego et qui n'appartiennent ni à la maison ni au phaspun. Tel est aussi le cas des personnes qui entrent dans les ordres et qui sont considérés comme ayant quitté maison et phaspun (voir Ramsay, 1890) ${ }^{20}$. Agu et machung quant à eux, tant qu'ils ne sont pas mariés ailleurs, peuvent toujours rejoindre le mariage de groupe.

\section{La génération Ego - 1}

Venons en maintenant à la génération Ego - 1 et faisons à nouveau un schéma (fig. 5). Les informateurs ont parfois du mal à répondre et cela vient probablement du fait que la terminologie évolue, putsha/pomo s'employant de moins en moins au profit de tshao/ tshamo voire de no/nono/coco/nomo ${ }^{21}$. Pour retrouver les termes de parenté c'est-à-dire grosso modo si c'est pomo/putsha $(\mathrm{P})$ ou tshao/tshamo $(\mathrm{T})$ ils raisonnent à l'envers :

- comment appelles-tu le fils de ton frère aîné ?

- je suis son agu donc je l'appelle tshao.

On voit donc que ce qui est déterminant pour la génération Ego - 1 est la génération Ego + 1 qui lui sert de référence. Comme quoi la série aba, ama, ane, azhang, agu, machung est plus stable que la série pomo, putsha, tshao, tshamo. Donc si Ego est masculin et s'il est l'agu ou l'azhang, son neveu, s'appelle tshao et tshamo. S'il s'appelle aba c'est pomo (bumo) et putsha (bu-tsha). On appelle donc pomo et putsha ses propres enfants puisque on est l'aba. 
Figure 5
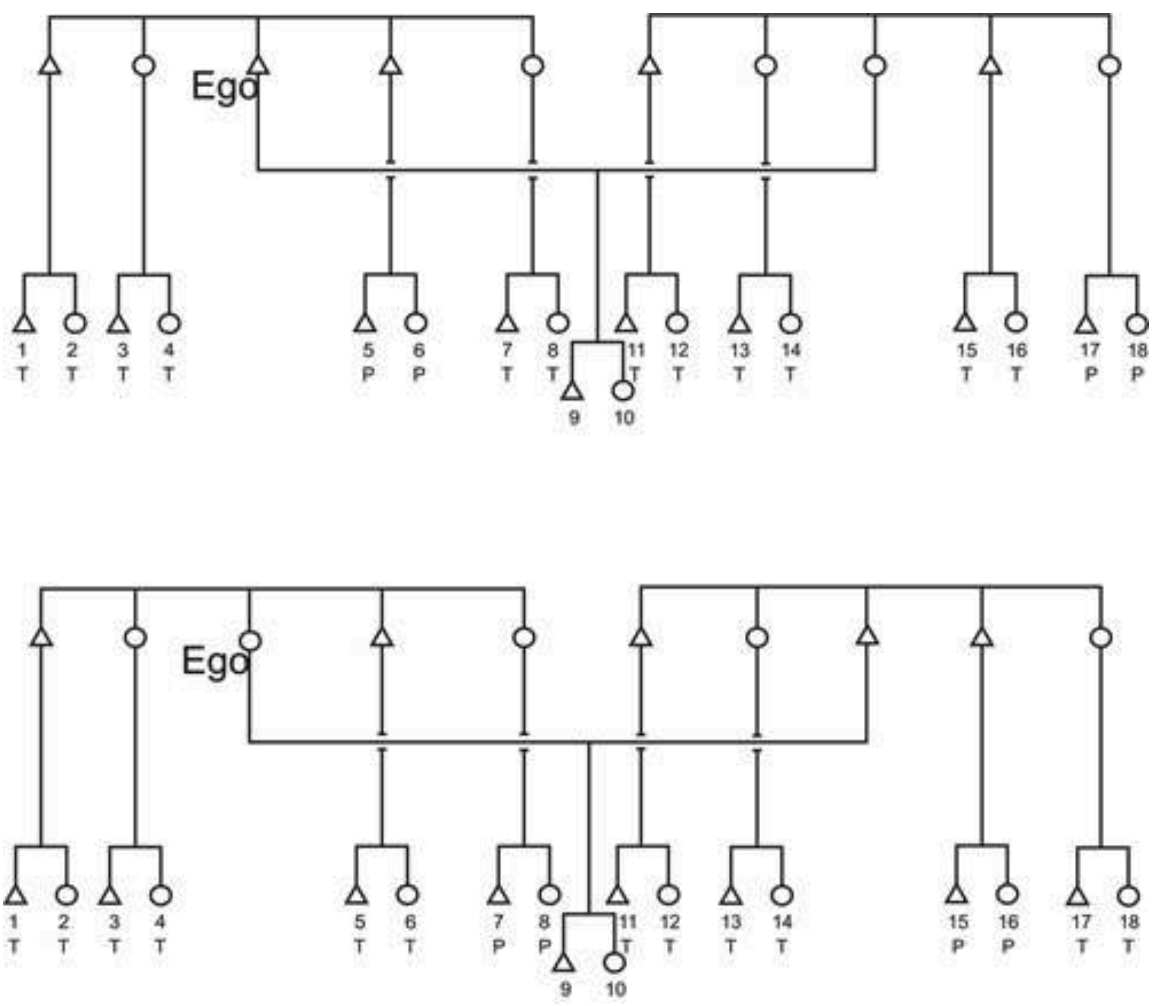

Ainsi Ego masculin est l'agu de 1 et 2 (puisqu'il est le jeune frère de leur père) et appelle 1 et 2 tshao et tshamo. Par contre il est l'aba de 5 et 6 puisqu'il est le frère aîné de leur père et donc il appelle 5 et 6 putsha et pomo. Bien entendu il appelle ses propres enfants 9 et 10 putsha et pomo. Les enfants des sœurs sont appelés tshao/tshamo de toute façon puisque Ego est azhang des enfants de sa sœur aînée et azhang des enfants de sa sœur cadette $(3,4,7,8)$.

Maintenant on connaît la règle comme quoi quelle que soit la façon dont ma femme appelle ses oncles et ses tantes je les appelle de la même façon et réciproquement. La règle est la même en ce qui concerne les neveux. Donc tous les termes d'adresse de 1 à 8 (et bien entendu 9 et 10) que nous venons d'énumérer sont aussi employés par le conjoint d'Ego. Pour les suivants de 11 à 18, il faut prendre le conjoint d'Ego comme repère, sachant que son mari utilisera les mêmes termes.

La femme d'Ego est l'ane de 11 et 12 qui sont donc tshao/tshamo pour la femme d'Ego (et donc pour son mari); elle est machung de 13 et 14 qu'elle appellera donc tshao/tshamo; elle est l'ane de 15 et 16 qu'elle appelle donc tshao/tshamo; enfin elle est l'ama de 17 et 18 qu'elle appelle donc putsha/pomo.

Précisons que ces appellations sont données suite au raisonnement que je viens de raconter, le plus souvent à haute voix. Mais dans la pratique on n'appelle plus ses neveux et nièces putsha et pomo. Même tshao/tshamo tendent à disparaitre au profit de nono/nomo. Tshao/tshamo résiste pour les petits-enfants et les petits-neveux.

Il va de soi que la tendance à remplacer pomo/putsha par tshao/tshamo est due à la déliquescence de la polyandrie. Tant que $\mathrm{F}$ et $\mathrm{FeB}$ étaient mariés à la même femme il était évident que tous leurs enfants étaient des « fils » et « filles ». 
51 De plus comme no/nono et nomo sont couramment employés, on tend aussi vers l'assimilation des générations - 1 et -2 aux cadets d'Ego.

Restons-en au système traditionnel de nomination des neveux et nièces. Ce système groupait ensemble sous le vocable de tshao/tshamo les enfants qui les appellent agu/ azhang/ane et machung. C'est-à-dire que ce qui est soigneusement distingué à la génération Ego + 1, les sœurs cadettes de mère (MyZ), les frères cadets de père (FyB), les sœurs de père $(\mathrm{FZ})$ et les frères de mère $(\mathrm{MB})$ se trouvent regroupés en deux vocables à travers leurs neveux.

53 Ceci n'est peut-être pas très étonnant si l'on admet que le fondement de la terminologie de parenté renvoie à un mariage de groupe entre des frères et des sœurs. Tous les enfants de ce mariage de groupe sont pomo et putsha, et tous les enfants des autres germains des tshao et tshamo. Mais cela inverse la question et nous renvoie d'Ego - 1 à Ego +1 . Si cette terminologie renvoie à un mariage de groupe entre des frères et des sœurs, elle pourrait se contenter de distinguer ceux qui font partie du mariage de groupe de ceux qui en sont exclus. Pourquoi la terminologie distingue-t-elle soigneusement dans ce qui est rejeté hors de ce mariage de groupe plusieurs termes? Plus précisément Pourquoi distingue-t-elle machung d'ane et agu d'azhang? Peut-être parce que machung et agu ne sont pas membres du mariage de groupe mais peuvent éventuellement le devenir. Et s'ils le deviennent on ne s'adresse plus à eux avec ces mots machung et agu: Grist (1979) fait remarquer que si un agu rejoint le mariage polyandrique, il devient aba. À la génération suivante, la question ne se pose plus. Ou bien ils ont rejoint le mariage de groupe ou bien ils ne l'ont pas fait. Ils n'a a donc plus que deux classes d'enfants et de neveux.

54 Ceci expliquerait peut-être pourquoi les moines et nonnes sont appelés azhang et ane et non pas agu et machung. Ils ont définitivement quitté maison et phaspun et ne sont pas potentiellement membre du mariage de groupe. Ils ne font partie ni de la maison, ni du phaspun $^{22}$.

55 Rappelons enfin qu'à la génération Ego - 2 tous les petits-enfants et petits-neveux peuvent être appelés tshao/tshamo. Ce ne sont pas pour autant des termes d'adresses généraux comme les huit du noyau de base quoiqu'il ne soit pas interdit à un vieil homme d'appeler un petit enfant qui n'est ni son neveu ni son petit-fils tshao. Mais dans ce cas là la métaphore est explicite. Le vieil homme veut exprimer ainsi une affection particulière pour cet enfant.

56 Une dernière remarque avant de passer à la nomenclature de désignation. On a souvent tendance à prendre les enfants comme référence. Un peu comme dans une famille française où le père dira à son enfant « demande à maman », puis finira par appeler luimême sa femme « maman ».

\section{Termes de désignation à Leh}

Voyons maintenant le tableau des termes de désignation. Les principales différences avec le système des termes d'adresse sont les suivantes : 
Figure 6

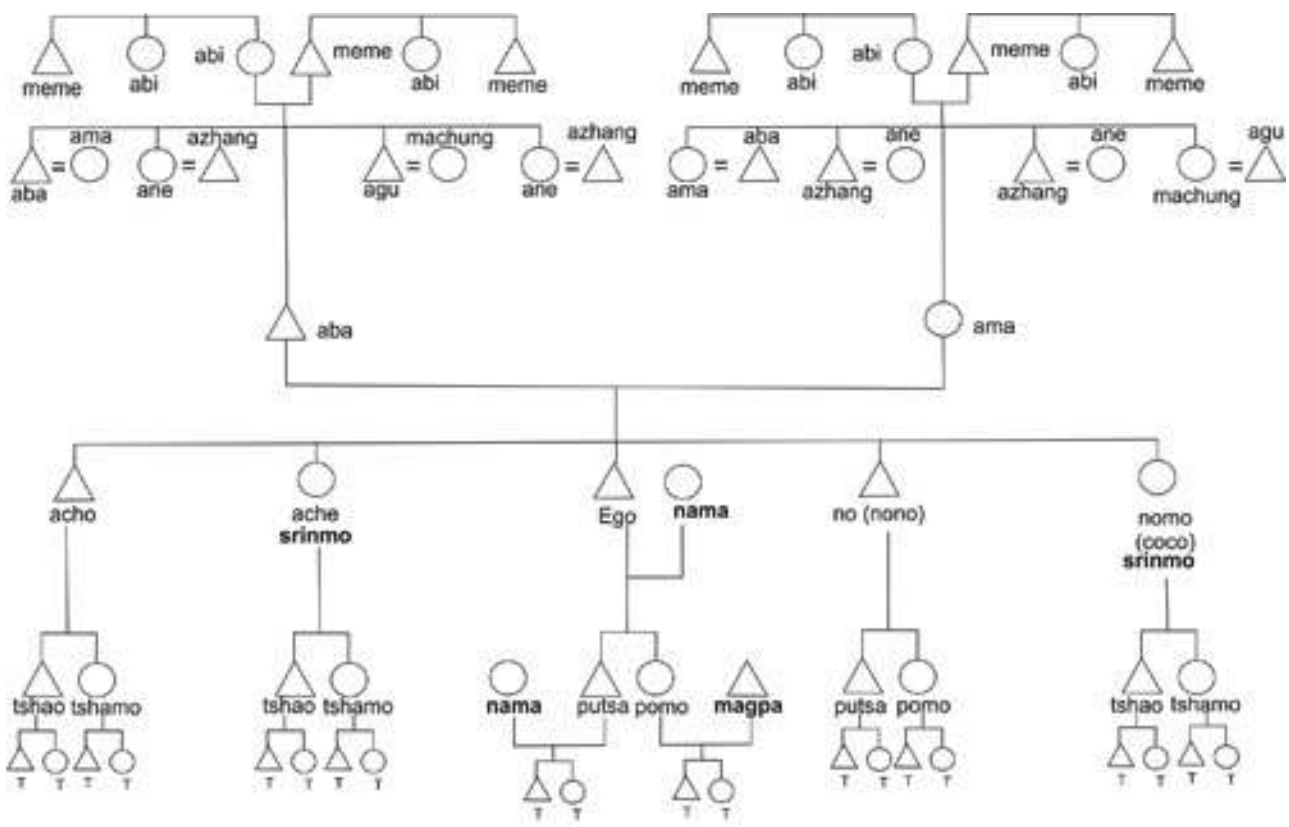

1) On signalera d'abord que certains informateurs ont du mal à désigner le neveu du côté du frère. Ils disent plus volontiers noe putsha, noe pomo, acoe putsha, acoe pomo : le fils/la fille de mon frère cadet/aîné. Par contre tous les enfants de mes sœurs sont des tshao/tshamo.

2) Nama ( $\left.m n a^{\prime}-m a\right)$ veut dire l'épouse, la femme et la belle-fille. Makpa (mag-pa) désigne le mari et le gendre. Voici donc deux autres termes transgénérationnels ${ }^{23}$. Pour le reste il n'y a pas de changement sauf en ce qui concerne les mots mingbo et sringmo.

3) En effet, nous dit Grist mingbo (ming-po) s'emploie pour dire frère lorsque le locuteur est une femme et sringmo (sring-mo) s'emploie pour dire sœur lorsque le locuteur est un homme. Il convient de préciser :

61 a) Effectivement une fille dira nye mingbo, "mon ou mes frères ", lorsqu'elle n'a pas besoin de préciser l'âge relatif. Elle emploiera plutôt mingbo au pluriel comme collectif «mes frères », les uns étant plus âgés d'autres plus jeunes. 
Figure 7

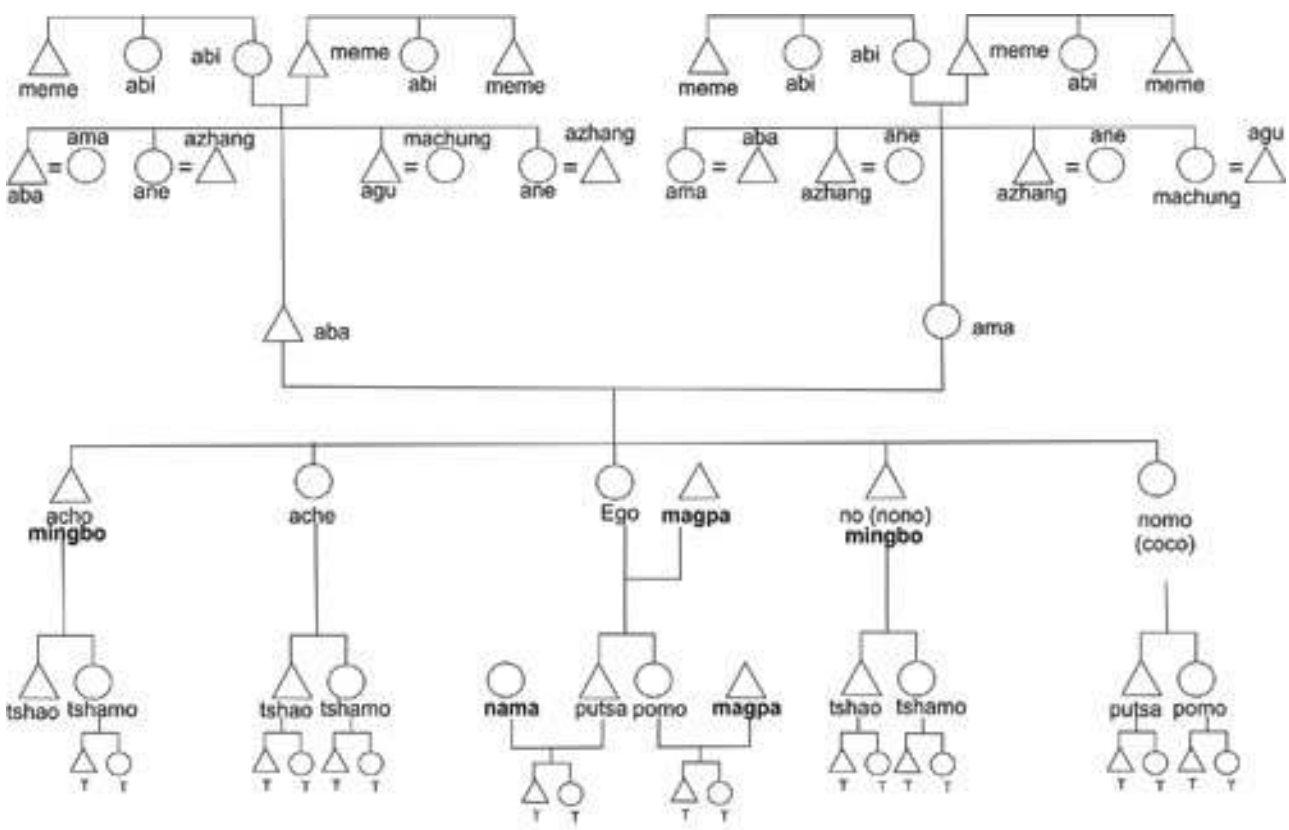

\section{Un garçon peut dire :}

nga(la) ache sum nomo nyis yot : j'ai trois sœurs plus âgées et deux plus jeunes.

Nga(la) sringmo snga yot : j'ai cinq sœurs

Nga(la) achenomo snga yot : même sens

Et une fille pourrait lui demander :

Nyerangi sringmo kane yot? : où est votre sœur?

Nyerangla sringmo tsam yot? : combien avez-vous de sœurs?

e) Le problème se pose plutôt pour les frères d'un garçon (ou on ne peut employer mingbo réservé aux frères des filles) et pour les sœurs d'une fille (où on ne peut employer sringmo réservé aux sœurs des garçons). S'il y en a plusieurs on peut toujours employer les collectifs acono et achenomo obtenus simplement en juxtaposant les deux mots. Mais imaginons qu'un locuteur masculin veuille parler à une fille de sa sœur sans savoir son âge relatif par rapport à son interlocutrice. J'ai remarqué que dans ces cas là 
on emploie plutôt le terme qui désigne l'aîné : ache (et réciproquement aco quand une fille parle).

f) Aujourd'hui sringmo tend à prendre le sens général de sœur et mingbo de frère, quelque soit le locuteur et l'âge relatif comme dans les langues indo-européennes. Mais, pris sur le fait, la plupart de ceux qui ont fait la «faute » la reconnaissent; comme quoi cette évolution est récente. L'ancien sens normatif de mingbo et sringmo reste conscient $^{25}$.

Pourquoi ces termes de sringmo et mingbo ? Il est possible de faire un rapprochement avec la génération d'Ego + 1 au niveau des termes d'adresse et de désignation. On a vu qu'on distingue les frères ainés/cadets du père mais pas les sœurs ainées/cadettes du père. De même sringmo est un collectif pour les sœurs d'Ego, mais pour le frère on continue à distinguer aco et no. Et on distingue les sœurs ainées/cadettes de la mère mais on ne distingue pas les frères ainés/cadets. Et de même mingbo est collectif pour tous les frères mais une personne du sexe féminin doit distinguer ache et nomo.

La question est ainsi déplacée : quand on parle des germains et des oncles et tantes de son propre sexe, on doit bien préciser l'âge relatif. Pour un homme aco/no, aba/agu; pour une femme ache/nomo, ama/machung. Quand on parle d'un germain ou d'un oncle ou tante du sexe opposé on peut s'en dispenser. Mingbo englobe aco/no et il y a azhang qui est un terme unique pour aîné et cadet de mère : sringmo englobe ache/nomo et ane est un terme unique pour aînée et cadette de père. On a alors une correspondance terme à terme.

Celui que mon père désigne comme aco je l'appelle $a b a$.

Celui que mon père désigne comme no je l'appelle agu.

Celles que mon père désigne comme sringmo je les appelle ane.

Celle que ma mère désigne comme ache je l'appelle ama.

Celle que ma mère désigne comme nomo je l'appelle machung.

Ceux que ma mère désigne comme mingbo je les appelle azhang.

Si cette comparaison est éclairante, elle ne résout pas tout. Pourquoi sringmo et mingbo en tant que termes de référence uniquement, alors que pour les oncles et tantes le raisonnement reste valable pour les termes de désignation aussi bien que les termes d'adresse, puisque ce sont les mêmes ? Peut-être parce qu'en adresse la génération, ou plus précisément le niveau (génération plus opposition aîné/cadet), prime tout.

71 Une dernière remarque. Les termes de désignation dépassent eux aussi largement le cadre strict de la parenté. Voici quelques exemples pris sur le vif.

- À l'entrée d'un monastère : «le moine (meme) est là ? - oui »

- À des enfants à l'entrée d'une réunion : «Va chercher un adulte (aco). Mais un adulte (aco chenmo) hein! (chenmo veut dire grand).»

- À une petite fille qui rentre de l'école : « La maitresse (ache) était-elle là ?»

On voit donc apparaître des termes de parenté là où en français on aurait " quelqu'un, un homme ", etc. Par exemple à quelqu'un qui était absent on peut dire : "un aco, un azhang, un $a b a$, etc. est venu te voir.» On prend alors l'âge de l'interlocuteur comme référence. 


\section{L'évolution de la terminologie}

73 1) La liste des termes d'adresse est stable. En gros la règle est donnée par Riaboff (car la règle est aussi valable au Zanskar) : « un Ego s'adresse à des affins par des appellations qu'il applique également à ses consanguins » (1997, p. 80). La liste est stable et son emploi évolue vers un renforcement de l'aspect générationnel à niveaux, les critères de la génération et de l'âge relatif tendent, avec le sexe, à être les seuls.

74 2) Tshao/tshamo évolue vers le sens indo-européen de neveu/nièce, ceci en adresse comme en désignation.

75 3) Les termes distinguant les futurs alliés, soit sringmo pour la sœur d'un frère qui joindra un autre phaspun et une autre khangpa et mingbo pour le frère d'une sœur, parce qu'elle joindra un autre phaspun et une autre khangpa, tendent, eux aussi, à disparaitre au profit d'un sens indo-européen de « frère » et « sœur ».

76 4) Comme pour l'adresse, pour la désignation la tendance générationnelle (renforcée par l'opposition aîné/cadet) se développe. De la même façon qu'on n'adresse plus systématiquement du aco à eZH, mais du aco ou du nono selon son âge relatif par rapport au locuteur, de la même façon évolue la façon d'en parler. Sauf pour la génération Ego +1 où cette évolution résiste.

77 5) De même la règle d'appellation réciproque entre mari et femme tend à disparaitre au profit du seul âge relatif. Sauf pour la génération Ego +1 où elle résiste.

78 La représentation traditionnelle que projette la terminologie de parenté est celle d'un mariage de groupe entre des germains et des germaines. Ce mariage est un mariage, en principe, entre les deux aînés. Les jeunes frères et les jeunes sœurs des deux aînés s'y ajoutent successivement sans cérémonie. C'est de là que découlait la règle d'appellation réciproque. Si Ego est masculin, sa femme est une aînée, non pas parce qu'elle est plus âgée que lui, mais parce qu'elle-même est l'aînée du groupe de sœurs ${ }^{26}$.

Évidemment la disparition de la polyandrie fraternelle et de la polygynie sororale expliquent cette évolution, ainsi que le déclin de l'institution du phaspun. On évolue vers un système générationnel (renforcée par l'opposition aîné/cadet) avec quelques traits indo-européens.

\section{Conclusions}

80

81 1) Tout d'abord on constatera qu'il reste encore un certain flou sur des points de détail. Je m'apprêtais à écrire «les recherches continuent». Elles continuent effectivement mais ce flou peut être éclairé sous un autre angle depuis la publication d'un article de Zeisler $^{27}$. J'avais choisi Leh parce que c'est l'ancienne capitale royale, l'actuel chef-lieu de district, bref la ville, la référence. En fait Zeisler démontre qu'on peut en gros distinguer deux groupes de parlers au Ladakh, qu'elle appelle kenhat (gyen-skad) et shamskat (gsham-skad). Or Leh est à la charnière des deux zones dans lesquelles sont parlés chacun des deux dialectes. Il faudrait refaire le relevé de la nomenclature de parenté dans un village du Haut-Ladakh, (kenhat), en amont de Leh, et dans un village du bas-Ladakh (shamskat) en aval de Leh, pour compléter cette étude. 
82 2) Une explication de ce modèle induit de mariage de groupe est peut-être à chercher dans les coutumes des Dardes bouddhistes ${ }^{28}$. On sait que les Ladakhi considèrent que leur civilisation relève en partie du Tibet et en partie des Dardes ${ }^{29}$, qu'elle constitue en quelque sorte un syncrétisme entre les deux. Or le mariage de groupe existe bel et bien chez les Dardes bouddhistes.

D'après Vohra ${ }^{30} 15 \%$ des mariages de la première génération, c'est-à-dire en gros de ceux qui avaient entre 60 et 70 ans lors de son enquête en 1980, donc qui sont nés vers 1910-1920, sont des mariages de deux frères avec deux sœurs, chaque frère épousant une sœur (p.140). Sept cas sur 45. Il doit s'agir d'une coutume ancienne car le pourcentage tombe à $4 \%$ pour la seconde génération (deux cas sur 46 ) et $8 \%$ à la troisième (trois cas sur 38).

Deux sœurs qui épousent chacune un frère, cela n'est théoriquement pas un mariage de groupe. Mais Vohra laisse entendre qu'il s'agit d'un mariage de groupe déguisé. Il en donne un exemple p. 88. Dans une famille il y a cinq frères. Les deux premiers ont épousé deux sœurs d'une famille $\mathrm{x}$, et les deux suivants deux autres sœurs d'une famille y. Et Vohra de préciser : «Informal marital relationships prevailed among the brothers and their wives, but the children born in the household were known as the children of the elder brother». Plus explicite encore est l'exemple de la page 85: "The two adopted sons received in common two sisters as wives from Chogolag-pa household. Though officially Horphel was married to Sonam Skid and Samdan to Sam, they both shared their wives. In any case the elder brother, Horphel, would be the only one to have the status of father and it would be his eldest son who would become the household head in the following generation ». À noter qu'il s'agit de deux frères adoptés, alors qu'un aurait suffi.

3) Ces traces de mariages de groupe décrites plus haut en citant Ramsay et Grist sont elles suffisantes pour affirmer l'existence d'un mariage de groupe à une certaine époque dont la nomenclature de parenté serait encore aujourd'hui le reflet? Peut-être pas. Mais il existe une autre façon de voir les choses. Nous allons voir que l'approche du cas ladakhi est finalement différente de celle du cas omaha étudié par Kohler.

L'unité de base de la société ladakhi est la maisonnée, la khangpa. Je dis maisonnée et non pas maison car la khangpa ne désigne pas nécessairement un bâtiment unique. Il peut y avoir les annexes, mais maison principale (khangchen; khang-chen) et annexe(s) (khangu ou khangchung; khang-chung) forment une seule khangpa. Habituellement (fautil le répéter je parle de la situation d'il y a bientôt 40 ans) vivent dans la maison principale le couple, ou le trio polyandrique, et leurs enfants non mariés. Les vieux parents se retirent dans une annexe après le mariage de leur aîné.

Dans l'organisation sociale du village seule, les khangpa sont des unités électives. Le chef du village est élu sur le principe d'une voix par khangpa. Il en est de même lors des réunions, à laquelle n'assiste qu'un représentant par khangpa. Il en est de même dans les rituels où certains rôles sont joués par un membre de chaque khangpa année après année à tour de rôle, etc. Bref il y a des individus mais il y a surtout des khangpa. Il existe néanmoins des groupes d'unifiliation patrilinéaires, les phaspun (pha-spun, pha père, spun, germain) qui ne remontent pas à un ancêtre commun fut-il fictif. Simplement la règle d'appartenance est la patrifiliation, ce qui est suffisant.

88 J'ai déjà démontrési que la maison ladakhi était un tout insécable. Et que pour introduire un nouvel élément (un nouveau né, une jeune mariée) ou au contraire 
retirer un élément (en cas de décès) il fallait « dissoudre » la maison dans le phaspun. La maison, groupe compact et insécable disparait, le phaspun, groupe d'individus, le remplace. Le nouveau-né, la jeune mariée, sont en tant qu'individus intégrés dans ce groupe d'individus qu'est le phaspun (ou le contraire en cas de décès) et, une fois les cérémonies terminées, la khangpa se reforme. Il n'est donc pas absurde de penser que, idéalement, je dis bien idéalement car cela ne renvoie pas à la réalité ethnographique, le mariage se fait entre deux maisons, ce qui implique effectivement l'union de toutes les sœurs avec tous les frères.

La nomenclature de parenté refléterait alors le mariage idéal non pas entre deux individus mais entre deux khangpa. Ceci expliquerait qu'il n'y a qu'un mariage par génération. Il était théoriquement exclu qu'un cadet se marie (sauf mariage uxorilocal). Les vieux parents se retiraient dans l'annexe, et tous ceux en âge de procréer qui n'entraient pas dans le mariage polyandrique ou polygynique étaient censés quitter la maison : moine, nonne ou mariage uxorilocal.

Dans cette logique l'un mariage par génération et par maison, les enfants du trio ou du quatuor polyandrique étaient considérés comme des fils de l'aîné. Il n'y avait d'ailleurs de cérémonie de mariage qu'avec l'aîné. Les cadets entraient ensuite dans le lit de leur belle-sœur sans autre forme de rituel. Il en était de même du mariage polygynique « en gendre »: le mariage n'avait lieu qu'avec l'aînée. Mais il était effectivement impossible d'affirmer que tous les enfants étaient les enfants de l'aînée. On procédait autrement : les enfants de la cadette n'étaient pas reconnus. Si l'aînée n'avait donné naissance qu'à des filles on procédait à un nouveau mariage en gendre, même si la cadette avait eu un garçon.

91 Un seul mariage par génération permet de conserver l'unité, la cohésion de la khangpa. La succession des générations est représentée par une ligne (gyut; brgyud). L'explication fonctionnaliste de ce système et qu'il a pour but de ne pas diviser la terre ; explication plausible mais insuffisante puisqu'il existe d'autres moyens de conserver le terroir intact.

92 4) Peut-on aller plus loin? Essayer de remonter dans le temps? Allen a conçu comme point de départ, peut-être à l'époque paléolithique, un modèle "tétradique » dans lequel deux générations (qu'il appelle paire et impaire) alternent, chaque niveau étant réduit à deux fois un frère et une sœur ${ }^{32}$. Au niveau un (impair) les deux frères échangent leur sœur. Au niveau deux (pair) la règle du mariage avec la cousine croisée s'applique. Et on recommence. Le schéma d'Allen (Fig. 8) aide à comprendre : il y a donc deux sexes, indiqués par des losanges (en fait des carrés dont les diagonales sont parallèles aux côtés de la page) et des carrés. Le schéma peut être lu avec les losanges indiquant les femmes et les carrés les hommes ou vice-versa. De ce point de vue il est strictement symétrique. Donc si, par exemple, Ego (le losange noir) est un homme, il épouse sa cousine croisée et sa sœur épouse son cousin croisé frère de l'épouse d'Ego. Il n'est pas difficile de démontrer que ce schéma se reproduit à l'infini la génération impaire qui suit celle paire d'Ego, est identique à celle qui précède. Ceci est marqué par le « rouleau » à droite et à gauche du schéma qui indique que l'on peut lire le schéma de haut en bas ou de bas en haut selon que l'on remonte générations dans le temps ou que, au contraire, on les suit dans leur succession.

93 Il y aurait eu dans ce monde une terminologie de parenté à huit termes qui correspondent aux huit losanges et carrés de schéma. 
Figure 8

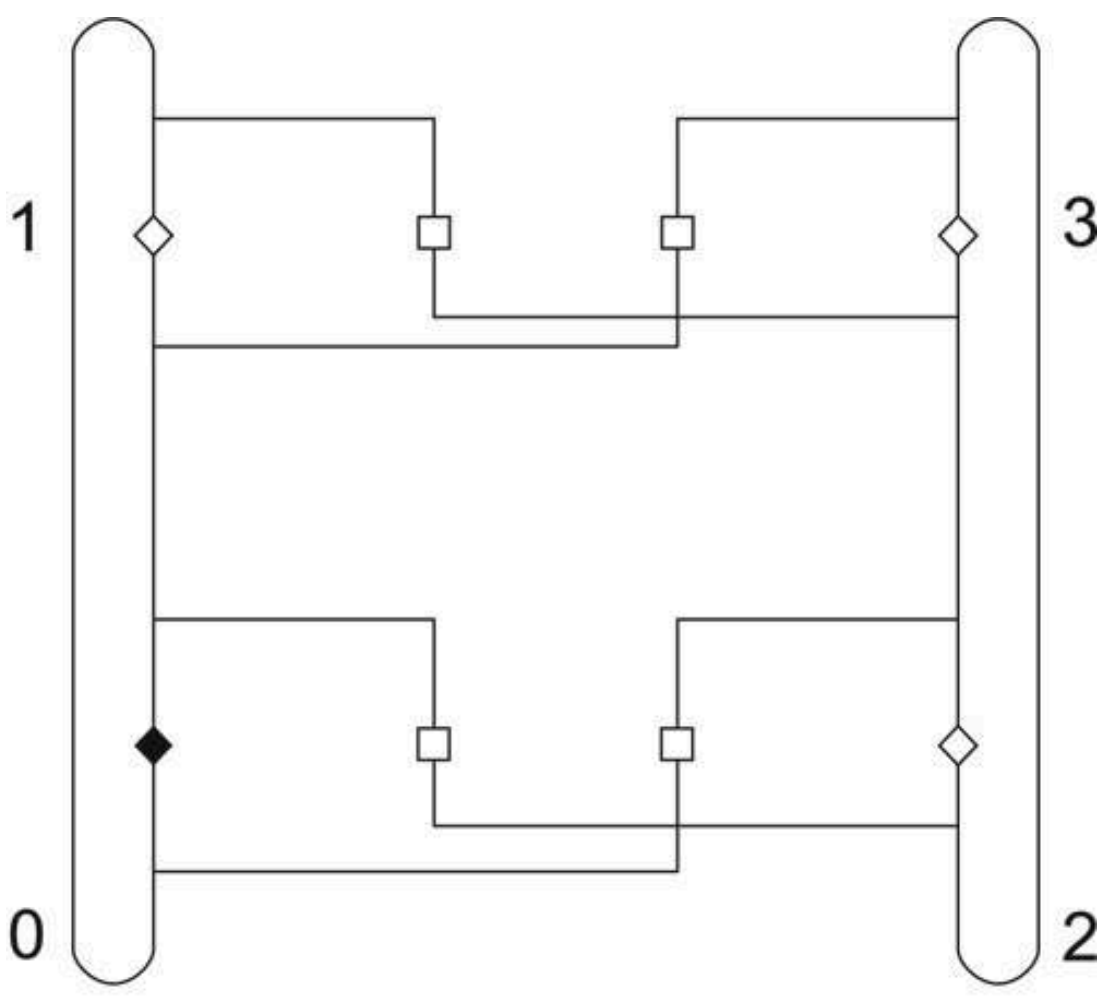

N. J. Allen

94 À la règle du mariage avec la cousine croisée s'ajoutaient deux autres règles. La première est l'assimilation des générations deux à deux. Ce qui fait qu'Ego, s'il est masculin, est assimilé à son grand-père et à son petit-fils $(\mathrm{FF}=\mathrm{SS})$; la seconde est l'équivalence de tous les frères $(F=F B)$ et sœurs $(M=M Z)$, etc. d'une génération.

D'après Allen, on peut construire toutes les nomenclatures actuelles en développant ce schéma selon trois règles diachroniques :

1) La disparition de l'identification entre générations alternées. FF devient différent des termes désignant des membres de la génération d'Ego et différent de SS. Ce qui est bien le cas au Ladakh.

97 2) La disparition des règles normatives (prescriptive), qui, permettent de fusionner en un mot un parent par le sang et un affin. C'est partiellement le cas au Ladakh.

3) La fin de l'assimilation des germains de même sexe et de même génération. C'est partiellement le cas au Ladakh.

Allen en donne lui-même un exemple à propos de la terminologie omaha ${ }^{33}$. Évidemment c'est loin d'être le cas dans le système ladakhi qui ne distingue pas les cousins croisés et parallèles et, de toute façon, interdit le mariage avec tout cousin. Bien entendu il serait toujours possible en appliquant les règles de dérivation de passer progressivement du système tétradique au système ladakhi. Mais une fois de plus c'est la génération Ego+1 qui attire l'attention. L'identité entre germains de même sexe et de même génération a été partiellement brisée en introduisant des termes spécifiques pour les cadets. Mais c'est le " partiellement » qui est intéressant.

Revenons au schéma d'Allen. Il est simple et clair parce qu'il n'y a qu'un garçon et une fille par couple et que chacun épouse le garçon ou la fille de l'autre couple. Mais que se 
passe-t-il lorsqu'il y a plusieurs enfants ? Allen a prévu le cas qui écrit ${ }^{34}$ que cela ne pose de problème que dans les sociétés qui séparent les générations (c'est-à-dire qui applique la règle de transformation № 1). Mais dans le système tétradique toutes les générations paires ne forment qu'une seule classe et, s'il y a, par exemple, plusieurs frères, et pas assez de cousines croisées à épouser, les frères surnuméraires peuvent toujours épouser quelqu'un de la génération Ego+2. Je sais bien que de tels systèmes existent réellement mais la systématisation qu'en fait Allen me gêne. D'abord dans cette société préhistorique on ne devait pas vivre bien vieux, et un mariage avec la génération +2 impliquait une forte différence d'âge mais surtout d'état physique. Ensuite rien ne dit qu'il y avait suffisamment de conjoints disponibles au sein de la génération $+2(\mathrm{ou}-2)$. Étant donné que tous les FB était des F classificatoires et toutes les MS des $\mathrm{M}$, il est plus logique de penser qu'on avait affaire à un mariage de groupe. Dans ce cas le schéma d'Allen tient parfaitement. Peu importe le nombre de frères et de sœurs, il n'y a même pas besoin de les représenter sur le schéma, un seul triangle et un seul petit cercle suffisent : ils ne représentent plus un seul individu, mais une série de frères et une série de sœurs. Nous ne sommes pas si loin du schéma ladakhi.

\section{BIBLIOGRAPHIE}

Aginsky, B. W.

1935 The mechanics of Kinship, American Anthropologist, XXXVII, pp. 450-451.

Allen, N. J.

1982 A Dance of relatives, Journal of the Anthropological Society of Oxford, 13, pp. 139-146.

1986, Tetradic Theory, an approach to Kinship, Journal of the Anthropological Society of Oxford, 17, 2, pp. 87-109.

2003 Tetradic theory and the origin of human kinship systems, in J. Lassèque (dir.), Émergence et évolution de la parenté (Paris, Éditions de l'ENS), pp. 44-54.

[2008] 2011 Tetradic theory and the origin of human kinship systems, in N. J. Allen, H. Callan, R. Dunbar \& W. James (dir.), Early Human Kinship, From sex to social reproduction (Chichester, WileyBlackwell), pp. 96-112.

2012 Tetradic theory and Omaha systems, in T. R. Trautmann \& P. M. White (dir.), Crow-Omaha : New Light on a Classic Problem of Kinship Analysis (Tucson, Presses de l'université de l'Arizona).

Augustins, G.

2000 À quoi servent les systèmes de parenté ? L’Homme, 154-155, avril-sept, pp. 573-598.

Crook, J. \& H. Osmaston (dir.)

1994 Himalayan Buddhist Villages, Environment, Resources, society and religious life in Zanskar, Ladakh (Université de Bristol).

Dendaletche, C \& P. Kaplanian (dir.)

1985 Ladakh, Himalaya Occidental, Recent Research $n^{\circ} 2$. Ethnologie, écologie. Actes du $2^{\mathrm{e}}$ colloque sur le Ladakh (Publiés comme numéro spécial de Acta Biologica Montana, $\mathrm{n}^{\circ} 5$, Pau). [Seconde édition (Paris-Pau) 2001]. 
Dumont, L.

1953 The Dravidian kinship terminology as an expression of marriage, Man, art. 54, pp. 34-39 ; repris dans Dumont, L. 1983 Affinity as a value, Marriage alliance in South India, with Comparative Essays on Australia (Chicago, Chicago university Press), p. 9. Version française dans Dumont, L. 1975 Dravidien et Kariera, L'alliance dans le mariage en Inde du Sud et en Australie (Paris, Mouton).

Goodenough, W. H.

1970 Description and Comparison in Cultural Anthropology (Chicago, Aldine).

Grist N., Farrington P., Phylactou M., \& P. Kaplanian

1977 Cambridge Undergraduate Ladakh Expedition (Cambridge).

1979 Reports in Ladakh, 1977-1979 (Cambridge).

Gutschow, K.

1995 Kinship in Zanskar : Idiom and Practice, in H. Osmaston \& P. Denwood (dir.), Recent Research on Ladakh $4 \& 5$, actes des $4^{\mathrm{e}}$ et $5^{\mathrm{e}}$ colloques sur le Ladakh tenus à Bristol en 1989 et à Londres en 1992 (School of Oriental \& African Studies, Londres), pp. 337-348.

Han Yi Feng

1937 The Chinese Kinship system, HJAS, 2, pp. 141-275.

1967 The Chinese kinship system (Havard University Press, Cambridge).

Hanlon, $\mathrm{H}$.

1893 Illustrated Catholic Missions (Londres).

Héritier, F.

1981 L'exercice de la parenté (Paris, Hautes Etudes/Gallimard/Le Seuil).

Kaplanian, P.

1981 Les Ladakhi du Cachemire (Paris, Hachette).

1991 Mythes et légendes sur les origines du peuplement du Ladakh, in E. Steinkellner (dir.)

Tibetan history and language. Studies dedicated to Uray Géza on his seventieth birthday (Wiener Studien zur Tibetologie und Buddhismuskunde 26, Arbeitskreis für Tibetische und Buddhistische Studien Universität, Vienne), pp. 255-270.

2008 Groupes d'unifiliation, parenté et société à maison au Ladakh (le phaspun), in M. Van Beek \& F. Pirie (dir.), Modern Ladakh; anthropological perspectives on continuity and change (Leyde, Brill).

Kirchhoff, P.

1932 Verwandtshaft Bezeichnungen und Verwandtenheitat, Zeitschrift für Ethnologie, LXIV, pp. 58, 46-49.

Kohler, J.

1897 Zur Urgeschichte der Ehe, Totemismus, Gruppenehe, Mutterrecht (Stuttgart, Entke) ; trad. anglaise par R. H. Barnes 1975 On the Prehistory of Marriage (Chicago, University of Chicago Press).

Kroeber, A. L.

1909 Classificatory systems of relationships, Journal of the Royal Anthropological Institute, 39 pp. 77-84 ; republié dans Kroeber, A. L. 1952 The Nature of culture (Chicago), pp. 175-181.

Lounsbury, F. G.

1959 Semantic Analysis of the Pawnee kinship usage, Language, 32, pp. 158-194.

Lowie, R. H.

1928 A Note on Relationship Terminologies, American Anthropologist, 30, pp. 263-267. 
Murdock, G. P.

1949 Social Structure (New York, Mac Millan). Trad. Française 1972 De la structure sociale (Paris, Payot).

Ourliac, P. \& J. de Malafosse

1968 Histoire du droit privé, III, Le droit familial (Paris, PUF).

Phylactou, M.

1989 Household organisation and marriage in Ladakh, Indian Himalaya, Ph. D. at the London School of Economics and Political Science (Université de Londres).

Pierre, de Grèce

1963 A Study of Polyandry (La Haye, Mouton).

Radcliffe-Brown, A. R.

1952 Structure and Function in Primitive Society, traduction française Structure et fonction dans la société primitive 1972 (Paris, Éditions de Minuit).

Ramsay, $\mathrm{H}$.

1890 Western Tibet, A practical Dictionary of the Language and Customs of the districts included in the Ladàkh Wazarat, Lahore, en ligne sur http://pahar.in/mountains/1890-western-tibet-dictionaryof-ladakh-by-ramsay-s-pdf/

Riaboff, I.

1997 Le Roi et le moine, figures et principes du pouvoir et de sa légitimation au Zanskar (Himalaya occidental), thèse de doctorat (Nanterre).

Sapir, E

1916 Terms of Relationship and the levirate, American Anthropologist, n. s., XVIII, pp. 327-337.

Shusky, E. L.

1965 Manual for Kinship Analysis (New-York, Holt Reinhard \& Wilson).

Vohra, R.

1989, An Ethnography of the Buddhist Dards of Ladakh, (Ettelbrück, Skydie Brown international).

Yuen Ren Chao

1956 Chinese Terms of Adress, Language, 1, janv.-mars.

Zeisler, B.

2011 Kenhat, the dialects of Upper Ladakh and Zanskar in Turin, in M. Turin \& B. Zeisler (dir.), Himalayan Languages and linguistics (Leyde, Brill).

Zimmermann, Fr.

1993 Enquête sur la parenté (Paris, PUF).

\section{NOTES}

1. Abréviations anglaises : $F$, father ; S, son; $Z$, sister ; D, daughter ; $B$, brother ; $H$, husband ; W, wife ; eB/eZ, elder brother/sister; $\mathrm{yB} / \mathrm{yZ}$, younger brother/sister. Ainsi par exemple FFZeS se lit « Father's father's sister's elder son », le fils aîné de la sœur du père du père (d'Ego).

2. Notons qu'on appelle un musulman kaga à la place de aco et quelquefois une musulmane didi concurremment à ache. Kaga étant le terme honorifique pour aco cela veut-il dire que l'on considère tous les musulmans comme des nobles? Il est plus vraisemblable de penser qu'il s'agit de la déformation de l'ourdou kaka, frère aîné, assimilé en kaga. L'emploi de didi (ourdou pour « sœur aînée »), concurremment à ache, le confirme. 
3. Il s'agit tout simplement du système canon. $C f$. Ourliac \& de Malafosse 1968, p. 84 et Héritier 1981, qui présente pp. 180-182 les différentes façons de calculer les degrés de parenté.

4. Cela rejoint une remarque plus générale : plus on est précis mieux c'est. On emploiera nyen ou nyendrung (gnyen ou gnyen-drung; parent au sens de kin and affine) par défaut, parce qu'on ne sait pas très bien la relation de la personne dont on parle, mais si on sait, agu ou machung, par exemple, c'est mieux que nyen. Et aco ou no c'est mieux que mingbo, ache ou nomo c'est mieux que sringmo, etc. (cf. infra).

5. L'intérêt des rtsima ne concerne que le mariage. Dans les années 1970 la sacro-sainte règle était qu'on ne pouvait épouser quelqu'un en deçà du $7^{e}$ degré, même si on pouvait relever déjà de sérieux coups de canif dans cette règle (Grist 1977, 1979 ; Kaplanian 1981 ; Phylactou 1989, etc.). $\mathrm{Au}$ Zanskar Gutshow (1995) parle du $5^{\mathrm{e}}$ degré et Riaboff du $7^{\mathrm{e}}$ degré du côté du père et du $4^{\mathrm{e}} \mathrm{du}$ côté de la mère (1997, p. 89).

6. Il est à noter que comme termes de désignation pomo/putsha veulent dire homme ou femme. On les emploiera pour dire « les femmes d'un côté et les hommes de l'autre » ou bien « il y avait 27 femmes et 30 hommes à la réunion ».

7. Murdock 1949 ; Shusky 1965, p. 22.

8. Kroeber 1909, pp.77-84, 1952, pp. 175-181. Dans ce texte célèbre Kroeber distingue huit critères, en l'occurrence : 1) la génération, 2) la différence entre ligne directe et collatérale, 3) la différence d'âge à l'intérieur d'une même génération 4) le sexe, 5) le sexe du locuteur, 6) le sexe de la personne par laquelle passe la relation de parenté, 7) la distinction entre parents par le sang et alliés, 8) le statut de la personne par laquelle passe la relation de parenté : vivante ou morte.

À cette liste Lounsbury (1956, p. 168 et n. 25) ajoute le critère de la différence entre les degrés de collatéralité (différences between kinsmen of varying degrees of collaterality). Un cousin germain peut être appelé d'une autre façon qu'un cousin au deuxième degré.

Murdock (1972) quant à lui, ajoute le critère de la polarité. « Il faut, dit-il, deux personnes pour qu'il y ait une relation de parenté.» "La reconnaissance de ce critère au niveau linguistique implique l'existence de deux termes de parenté distincts pour toute relation, chacune des personnes intéressées en employant l'une pour désigner l'autre. " Mais deux personnes peuvent aussi se désigner par le même terme. C'est le cas de cousin en anglais (1972, pp.111-115). Il appelle le critère 6 « bifurcation » (forking).

Quant à moi je fais remarquer qu'il existe des termes de parenté honorifiques, pour s'adresse aux membres de la noblesse et du haut clergé.

9. Lowie 1928, pp. 263-267.

10. Françoise Héritier (1981, p. 19) mentionne ces quatre cas et fait remarquer que Lowie ne cite pas tous les cas possibles mais tous les cas effectivement rencontrés dans le corpus ethnographique. Il reste encore un cas théorique : les frères de mère s'appellent comme le père, tandis que les frères de père sont affublés d'un terme différent.

11. Han Yi Feng 1937. Voir aussi Han-Yi-Feng 1967 et Yuen Ren Chao 1956.

12. Dumont note bien l'existence, dans les langues dravidiennes de l'Inde du Sud, de préfixes pour démarquer par exemple $\mathrm{F}$ de $\mathrm{FeB}$ et $\mathrm{FyB}$, mais il considère que des préfixes ne doivent pas être pris en compte et qu'il y a bel et bien « assimilation » (merging) des FB ou F. Je ne parle pas un mot de tamoul, mais doit-on considérer qu'en français la différence entre père, grand-père et beau-père ne doit pas être prise en compte? Dans ce cas que faire de la synonymie entre bellefille et bru, beau-fils et gendre?

De toute façon la question ne se pose pas en ladakhi au moins pour agu. Par contre si on refuse de prendre en considération machung, en tant que mot composé, toute la démonstration de cet article s'effondre. Or la symétrie entre agu et machung est évidente, et l'un est composé pas l'autre. Dumont 1953 pp. 34-39, repris dans Dumont 1983, p. 9. Version française dans Dumont 1975. Même position chez Augustins 2000, pp. 573-598. 
13. Cité par Zimmermann 1993, p. 49. Voir aussi dans le même ouvrage p. 125 : Selon RadcliffeBrown (1952, p. 70) «Ego perçoit du dehors la lignée patrilinéaire de sa mère dans son unité et applique à tous les membres de même sexe de cette lignée le même nom de parenté sur au moins trois générations.» Cette théorie n'est effectivement intéressante qu'à partir du moment où non seulement $\mathrm{M}=\mathrm{MZ}$ mais aussi $\mathrm{Z}=\mathrm{MZD}$ et $\mathrm{MMZ}=\mathrm{MM}$ comme c'est effectivement le cas chez les Iroquois (ibid., p. 50). Et p. 133 «Mais Radcliffe-Brown allait plus loin en disant qu'elles sont traitées "comme" la mère parce qu'elles appartiennent au même groupe de filiation patrilinéaire.» Voir aussi les critiques très sévères de Murdoch, op. cit., pp. 129-130.

14. Kohler 1897 ; trad. Anglaise 1975. Cité par Zimmermann (1993, pp. 124-125).

15. Sapir 1916, pp.327-337 ; Kirchhoff 1932, pp. 58, 46-49. Cet article propose aussi les quatre catégories de Lowie. B. W. Aginsky (1935, pp. 450-451).

16. Ramsay 1890.

17. Ce renforcement de la symétrie entre mariages makpa (uxorilocaux) et bagma (virilocaux) par la terminologie de parenté est corroboré par le discours des Ladakhi qui insistent sur la symétrie entre les deux cérémonies de mariages, les rôles du fiancé et de la fiancée étant tout simplement inversés. Le makpa «fait la fille ». Ce discours est peut-être théorique (je n'ai jamais assisté à un mariage makpa) mais il existe.

18. Hanlon 1893, p. 103. Je tiens à remercier John Bray de m'avoir envoyé une copie des textes introuvables de Hanlon. Le phortsak est aussi mentionné par Pierre de Grèce (1963) et par Grist (1979).

19. Gutschow (1995, p. 345) nous apprend à propos du Zanskar : « If childress, polyandrously wed brothers may bring in a second and even a third wife, usually related to the first wife. " Voilà qui pourrait être une confirmation ethnographique à condition que la nomenclature zanskari de parenté soit comparable avec celle du Ladakh proprement dit.

20. Notons que meme peut s'employer pour un moine et ane peut s'adresser à une femme célibataire assimilée à une nonne. Ane peut aussi désigner - c'est un comble - une femme facile, une femme illégitime voire une prostituée.

21. À ce propos j'ai remarqué que les femmes étaient beaucoup plus à l'aise dans la terminologie de parenté. Plusieurs fois le mari a appelé sa femme au secours laquelle répondait du tac au tac. Fils du frère aîné ? tshao! Fille du frère aîné ? tshamo !, etc.

22. Il arrive qu'un moine se défroque, et même qu'il se marie après. Cela ne change rien au raisonnement « théorique » auquel renvoie la terminologie de parenté.

23. On notera l'existence de deux termes bagma (bag-ma) et nama (mna'-ma) pour désigner la fiancée et l'épouse. Bagma désigne avant tout la future femme pendant la cérémonie du mariage (mais nama peut être utilisé), lequel s'appelle bagston. Par contre le fiancé et le mari (et le gendre) sont tous deux makpa (mag-pa). Ceci s'explique aisément par le changement de statut de la femme qui se déplace, change de maison et, il n'y a pas si longtemps que cela, de phaspun (groupe exogamique). Bagpo ou pagpo existe. Je l'ai entendu quelquefois.

24. En d'autres termes il ne s'agit pas du critère 5 de Kroeber mais du $6:$ «) Le sexe du locuteur Cette catégorie ne joue pas en anglais ni dans la plupart des langues européennes, mais on sait son importance dans nombre d'autres langues. Père, mère, frère, sœur et parents plus éloignés peuvent être nommés d'une certaine façon par un homme et d'une façon différente par sa sœur. 6) Le sexe de la personne par laquelle passe la relation de parenté - L'anglais n'exprime pas cette catégorie, aussi trouvons-nous souvent nécessaire de préciser si un oncle est un frère du père ou un frère de la mère, et si une grand-mère est paternelle ou maternelle. " Traduction Zimmermann, 1993, p. 106. Lequel Zimmermann précise en note : «Ce sixième critère, le sexe du parent intermédiaire, fut baptisé bifurcation par Lowie en 1928. Ce qui est exactement en jeu dans la bifurcation, comme le note Ward H. Goodenough, 1970, p.115, n. 18, ce n'est pas le sexe du parent intermédiaire mais la présence ou nom d'une différence de sexe entre, d'une part, le parent intermédiaire et, d'autre part, le plus âgé des deux individus dont la relation de parenté 
est à définir. Par exemple, soit $\mathrm{A}$ le neveu ou la nièce, $\mathrm{B}$ le parent intermédiaire (son père ou sa mère), $C$ l'oncle ou la tante : il y a bifurcation, s'il y a une différence de sexe entre $B$ et $C$, la paire de parents de même génération (coeval pair). » Évidemment ce raisonnement ne tient pas ici. Le locuteur, son interlocutrice et le frère (mingbo) de celle-ci, ou bien le locuteur, son interlocuteur et la sœur (sringmo) de celui-ci peuvent très bien être de la même génération. Faut-il diviser le critère 6 de Kroeber en deux sous-cas?

25. Sringmo et mingbo ne sont donc pas des termes de parenté au sens strict mais plutôt des collectifs. $Y$ en a-t-il d'autres?

- Il n'y a pas de mots pour « parents ». On juxtapose abaama comme on peut juxtaposer acono ou achenomo. On emploie quelquefois phama.

- Il n'y a pas non plus de termes pour les grands-parents. Selon la même technique on juxtapose abimeme.

- Maintenant si acono et achenomo peuvent désigner respectivement les frères ou les sœurs pour l'ensemble des frères et sœurs on utilise mingsring (mingbo + sringmo). Évidemment aconoachenomo aurait été un peu long. Mais alors que mingbo désignait les frères de sœurs et sringmo les sœurs de frères, mingsring s'emploie pour tous. D'autre part il peut parfois s'étendre aux cousins de $1^{\text {er }}$ degré au moins. On emploie parfois l'expression mingsringmingbo, dont il n'est pas sûr qu'elle soit strictement synonyme de mingsring.

Mingsring a un concurrent: spunla. Mais spunla est ambigu. Il n'y a qu'une minorité d'informateurs pour dire qu'il est strictement synonyme de mingsring et une majorité pour le traduire par parents par le sang (kin). Spunla s'oppose alors à nyen qui sont les parents au sens large, parents par le sang et alliés (kin and affines). Au Zanskar le terme désigne les consanguins (kin) uniquement, d'après Gutshow (1995) et d'après Riaboff gnyen-drung désigne au Zanskar aussi les parents au sens large, les parents par le sang étant sha khrag gnyen drung (1997, p. 89 et n. 32).

- Sur le même modèle que acono, mingsring, etc. il existe makpanama pour désigner le couple. Par contre il existe un mot pour époux/épouse c'est zhitmat. On peut poser la question à un homme et à une femme (ce n'est pas très poli, mais c'est grammaticalement correct) :

Zhitmat chachen? Vous êtes mariés, vous êtes un couple?

Et on répond

Nazha mingsring inok: (Non) nous sommes frères et sœurs

Ou bien

Nazha zhitmat inok (Oui) nous sommes époux

Il n'existe pas de termes généraux pour oncles, tantes, oncles et tantes, et cousin est désigné par aco, no, ache, nomo comme nous le savons maintenant. Il existe donc très peu de termes collectifs : mingbo, sringmo, zhitmat, sans oublier thrugu (phru-gu; enfants). Le reste est obtenu par juxtaposition de deux mots.

26. Je tiens à remercier Isabelle Riaboff pour ses commentaires fort utiles et pour m'avoir envoyé des informations qui ne figurent pas dans sa thèse.

27. Zeisler 2011.

28. Je remercie Bettina Zeisler de m'avoir suggéré ce rapprochement avec les Dardes lors de la présentation de ce texte dans le cadre du $13^{\mathrm{e}}$ Congrès de l'IATS (International Association of Tibetan Studies) à Oulan-Bator en juillet 2013.

29. Kaplanian 1991.

30. Vohra 1989.

31. Kaplanian 2008.

32. Une première esquisse dans Allen 1982. Le principal texte est Allen 1986. La première partie est reprise de façon plus détaillée dans Allen 2003 et, de façon encore plus étayée, dans Allen 2011.

33. Allen 2012.

34. Allen 2011, p. 106. 


\section{RÉSUMÉS}

L'un des traits caractéristiques de la terminologie de parenté au Ladakh est l'existence de mots différents de $\mathrm{M}$ et $\mathrm{F}$ pour MyZ et FyB alors que MeZ et FeB s'appellent comme M et F. Cette constatation met à mal les théories de l'anthropologie anglaise qui fait dériver la terminologie de parenté de l'existence de groupe exogamiques, mais renforce par contre celle de Kohler pour qui la terminologie est liée à l'idée de mariage de groupe. L'auteur montre que le mariage de groupe ladakhi est néanmoins assez différent du mariage de groupe omaha étudié par Kohler. Quelques lignes sont aussi consacrées au modèle tétradique d'Allen.

One of the characteristics of kinship terminology in Ladakh is the existence of terms for Mother's younger sister and Father's younger brother that differ from Mother and Father, while Mother's elder sister and Father's elder brother are called by the same term as Mother and Father respectively. This system challenges theories from English anthropology that claim that kinship terminology is derived from the existence of exogamous groups but supports Kohler's theory that kinship terminology is linked to the idea of group marriage. The author shows that Ladahki group marriage is nevertheless significantly different from the group marriage studied by Kohler. A few lines are devoted to Allen's tetradic model.

\section{INDEX}

Mots-clés : parenté, nomenclature, terminologie, théorie tétradique, Ladakh, Leh

Keywords : kinship, terminology, tetradic theory, Ladakh, Leh

\section{AUTEUR}

\section{PATRICK KAPLANIAN}

Chercheur indépendant, il travaille sur le Ladakh depuis 1975. En 1981, il a publié une monographie sur les Ladakhi. En 1985, il a organisé le second colloque sur le Ladakh. Il a publié depuis de nombreux articles.

kaplanianpatrick@hotmail.com 